To cite this article:

Chris Green, Liz Anderson, Sundari Joseph, Angus McFadyen \& Scott Reeves (2015) Expanding interprofessional research in the United Kingdom: A new national research Group. Journal of Interprofessional Care, 29:5, 407-408. DOI:

10.3109/13561820.2015.1081803

To link to this article: http://dx.doi.org/10.3109/13561820.2015.1081803

\title{
Expanding interprofessional research in the United Kingdom: A new national research group
}

\section{Introduction}

A recent review of interprofessional education in the UK invited CAIPE to instigate discussions amongst organisations and researchers for the purpose of "establishing a group to conduct an up-to-date, broad-based worldwide systematic review of evidence for pre-qualifying interprofessional education in health and social care" (Barr, Helme and D'Avray 2014: p.7). Such a challenging brief led CAIPE to consider the present state of interprofessional research and scholarship amongst CAIPE's membership and contemplate how this work could be co-ordinated and nurtured, how research capacity could be advanced, and how CAIPE could support the research agenda moving forward. In this editorial we articulate CAIPE's initial steps in this endeavour. We provide details of CAIPE's newly-formed research advisory group, set the scene based on a preliminary information gathering exercise and outline the group's intended activities to facilitate expansion of interprofessional research in the UK.

\section{The CAIPE Research Advisory Group}

Following up on the recommendations outlined in Barr et al.'s (2014) review, a small national research advisory group of UK-based researchers, brought together by a common commitment to enhancing and supporting interprofessional research, was convened. Its provisional remit: to enhance CAIPE's excellent scholarly reputation, formalise CAIPE's existing interprofessional scholarship activities, and provide a more prominent focus for research and evaluation across the CAIPE membership. The group's initial work has sought to collect information about the types of research activities that are undertaken in the institutions represented by CAIPE members. 
Furthermore, the group wanted to elicit members' perceptions of the support that CAIPE might provide in the future. To these ends, a survey was designed for online distribution. Questions were focused on garnering information on current/recent research and evaluation activity that respondents are involved in; and respondents' perspectives on the support needs they felt would most effectively strengthen recent and future research and evaluation activity. The survey was sent electronically to CAIPE's seventy Individual and Corporate Members.

Analysing the responses to the survey indicated that the overwhelming majority of current research and evaluation activity was local and unfunded with relatively little funded single or multi-site research and evaluation. Local, unfunded work tended to be small-scale qualitative evaluations of interprofessional learning in educational settings. Funded single and multi-site research, whilst limited, tended to be financially supported by regional divisions and local workforce partnerships responsible for planning and commissioning health education. In addition, respondents provided several suggestions related to provision that could enhance current and future research and evaluation, these included:

\section{Resources:}

- A repository of evidence-based interprofessional learning initiatives for sharing good practice

- A repository of key contacts that can be approached to provide research supervision and research mentorship (though the scope of these activities were not defined);

- A repository of research questions that the interprofessional community most urgently need to respond to;

- A repository of research/evaluation tools (with context-specific case studies) so that independent projects and evaluations could be more easily compared

\section{Networks:}

- Networking as enablers to join bids together for larger amounts of funding for multi-site research; 
- Networking to make links with related research outside of interprofessional learning specifically e.g. service improvement and quality enhancement.

Funding:

- Identification of funding sources for interprofessional research and evaluation

- Circulation of potential funding calls especially in relation to collaborative projects across sites.

- A peer review service for funding bids

Resources, networks and funding form the foundations of successful research capacity building and sustaining these conditions will challenge us as they have challenged others in this enterprise (e.g. Thomas and While, 2001; White, 2012; Thistlethwaite and GRIN Working Group, 2013).

\section{Future plans}

The Research Advisory Group, under the auspices of CAIPE, hopes to respond to these challenges in a meaningful way, by facilitating the conditions for the interprofessional community of practice in the UK to more actively engage as producers and users of research evidence. Cooke (2005) identifies six principles to building research capacity: skills and confidence; ensuring the research is "close to practice" (p.5); developing linkages and partnerships; developing appropriate dissemination; investments in infrastructure and building elements of sustainability and continuity. These principles are linked directly to the recommendations made by survey respondents - the need for resources, networking and funding. With limited funds the inteprofessional research community will need to more strategically draw upon the resources currently at its disposal. In practical terms the Advisory Group plan to:

- Provide a virtual space on the CAIPE website dedicated to interprofessional research. This will house repositories of evidence-based interprofessional initiatives and research tools; list key contacts to gather a community of research expertise who can provide research supervision and mentorship; and signpost the most pressing research agendas. This is aimed at sharing 
the best available practice, developing research skills, advocating networking opportunities across the interprofessional community and prioritising areas where research evidence is most needed.

- Facilitate networking through presence at interprofessional events. The success of research capacity building is dependent on engaging the people and organisations that constitute the interprofessional community. The establishment of the group was recently announced at the biennial John Horder lecture in London. We plan to nurture links further at the CAIPE Chair's event to be held in London on 6 November, inviting participants to register their interprofessional research interests and nurture the conditions for collaboration. Plans are also in progress to deliver a pre-conference workshop at All Together Better Health 8 to be held in Oxford in September 2016, expanding the interprofessional research community, linking research and practice teams and cultivating associations with international colleagues.

To optimise sustainability, we will actively explore further funding opportunities to support interprofessional research. The development of themed special interest groups as the network emerges and develops may allow wider investment opportunities to be identified and realised. There may be scope too for scheduling more regular research events that disseminate evidence and exchange knowledge.

\section{Concluding comments}

The group's initial activity has highlighted the need to think more strategically about interprofessional research in the UK. The current picture illuminates the valuable work going on within local settings, though the capacity and opportunity for developing larger scale, multi-site projects appears limited. There is a need for a coordinated research agenda that responds to the most pressing needs as we move towards an era of integrated health and social care. Such activity cannot be achieved in isolation, but through the concerted and continued commitment of the interprofessional community working together across organisational and disciplinary boundaries. This is an endeavour fraught with challenges. But we hope that a modest contribution in capacity building through the activities described in this editorial can lead to a more ambitious expansion of rigorous and relevant 
interprofessional research that can benefit service users and communities in the future.

\section{Declaration of interest}

The authors confirm that they have no interests to declare.

\section{References}

Barr, H. (2002). Interprofessional education: Today, yesterday and tomorrow. Occasional Paper No.1. London: The Higher Education Academy, HSP.

Barr H, Helme M \& D'Avray L (2014) Review of interprofessional education in the United Kingdom. Fareham: CAIPE.

Cooke, J (2005) A framework to evaluate research capacity building in health care. BMC Family Practice, 6, 44, doi: 10.1186/1471-2296-6-44.

Thistlethwaite, J. \& GRIN Working Group. (2013). Introducing the Global Research Interprofessional Network (GRIN). Journal of Interprofessional Care, 27, 107-109.

Thomas P and While A (2001) Increasing research capacity and changing the culture of primary care towards reflective inquiring practice: the experiences of the West London Research Network (WeLReN) Journal of Interprofessional Care; 15, 2 : 133-139.

White E (2012) Developing the Allied Health Professions Research Network: building research capacity and capability. International Journal of Therapy and Rehabilitation; 19, 11: 601-602. 